\title{
Universiteit
}

Leiden

The Netherlands

\section{Psychophysiological evidence for cortisol-induced reduction in early bias for implicit social threat in social phobia.}

Peer, J.M. van; Spinhoven, P.; Roelofs, K.

\section{Citation}

Peer, J. M. van, Spinhoven, P., \& Roelofs, K. (2010). Psychophysiological evidence for cortisol-induced reduction in early bias for implicit social threat in social phobia.

Psychoneuroendocrinology, 35, 21-32. Retrieved from https://hdl.handle.net/1887/15129

Version: $\quad$ Not Applicable (or Unknown)

License: $\quad$ Leiden University Non-exclusive license

Downloaded from: https://hdl.handle.net/1887/15129

Note: To cite this publication please use the final published version (if applicable). 


\title{
Psychophysiological evidence for cortisol-induced reduction in early bias for implicit social threat in social phobia
}

\author{
Jacobien M. van Peer ${ }^{a, *}$, Philip Spinhoven ${ }^{\text {a,b }}$, Karin Roelofs ${ }^{a, c}$ \\ a Leiden University Institute for Psychological Research, Department of Clinical, Health and Neuropsychology, \\ Leiden University, PO-Box 9555, 2300 RB Leiden, The Netherlands \\ ${ }^{\mathrm{b}}$ Department of Psychiatry, Leiden University, PO-Box 9600, 2300 RC Leiden, The Netherlands \\ ' Leiden Institute for Brain and Cognition (LIBC), PO-Box 9600, 2300 RC Leiden, The Netherlands
}

Received 15 June 2009; received in revised form 11 September 2009; accepted 11 September 2009

\section{KEYWORDS}

Social anxiety disorder;

Cortisol;

Event-related potentials;

Emotional Stroop;

Angry face;

Threat bias

\begin{abstract}
Summary The stress hormone cortisol is important for the regulation of social motivational processes. High cortisol levels have been associated with social fear and avoidance, which play an important role in social anxiety disorder (SAD), as does hypervigilant processing of social threat. However, causal effects of cortisol on threat processing in SAD remain unclear. In an event-related potential (ERP) study we investigated the effects of cortisol on task-irrelevant (implicit) processing of social threat in SAD, exploring the temporal dynamics as well as the role of symptom severity and stimulus awareness. Angry face processing was measured in participants with clinical SAD after double-blind, within-subject oral administration of cortisol $(50 \mathrm{mg})$ and placebo, using a masked and an unmasked emotional Stroop task. Both tasks showed significantly increased P2 midline ERP amplitudes for angry compared to neutral and happy faces in the placebo condition, reflecting an early attentional bias for social threat in SAD. Furthermore, cortisol administration significantly decreased P2 amplitudes for masked angry faces. This effect correlated with social anxiety, showing stronger decreases in patients with higher levels of social anxiety. These results indicate a highly specific effect of cortisol on early motivated attention to social threat and, together with previous findings, highlight the importance of motivational context (stimulus- or task-relevance) as well as symptom severity.
\end{abstract}

(C) 2009 Elsevier Ltd. All rights reserved.

\footnotetext{
* Corresponding author. Tel.: +31 71 5273835; fax: +31 715274678.

E-mail address: jpeer@fsw.leidenuniv.nl (J.M. van Peer).
}

\section{Introduction}

Social anxiety disorder (SAD, or social phobia) is characterized by intense fear and avoidance of social situations. Cognitive models of SAD suggest that it is associated with biases in attending to threat-related information, and that 
these information-processing biases may be implicated in the development and maintenance of anxiety symptoms (see e.g., Clark and Wells, 1995; Rapee and Heimberg, 1997; Mobini and Grant, 2007; Schultz and Heimberg, 2008). There is, however, no consensus about the specific processing stages in which these biases occur. In the present study we aim to gain more insight in the temporal dynamics of biased processing of social threat in SAD, by investigating event-related potentials (ERPs) during masked and unmasked versions of a pictorial emotional Stroop task. In addition, recent neurobiological accounts propose that increased social fear and avoidance as found in SAD may be related to high levels of the stress hormone cortisol (e.g., Condren et al., 2002; Hermans and van Honk, 2006; Roelofs et al., 2009). However, causal effects of cortisol on threat processing remain unclear. Therefore, our second purpose was to investigate the effect of cortisol administration on social threat processing in SAD.

Although a wide range of studies has provided empirical evidence for biased processing of social threat in SAD (see e.g., Bar-Haim et al., 2007; Mobini and Grant, 2007 for reviews), results are conflicting with regard to the direction of this bias. Several studies have found a bias towards threat (or threat vigilance) in socially anxious participants, as indicated by longer reaction times (RT) for color-naming threat vs. neutral stimuli in emotional Stroop tasks (e.g., Mattia et al., 1993; Amir et al., 2002; Spector et al., 2003) or speeded responses to threat cues in spatial attention paradigms such as dot-probe or visual search tasks (e.g., Asmundson and Stein, 1994; Gilboa-Schechtman et al., 1999; Mogg and Bradley, 2002; Musa et al., 2003; Mogg et al., 2004). Other studies, however, reported a bias away from threat (or threat avoidance) in socially anxious participants, as indicated by shorter RTs for threat vs. neutral stimuli in emotional Stroop tasks (Putman et al., 2004) or longer RTs to threat cues in dot-probe tasks (e.g., Mansell et al., 1999; Chen et al., 2002). Although these discrepant findings may be in part related to variation in experimental paradigms (e.g., Stroop vs. dot-probe) or stimulus materials (words vs. face pictures) (see Bar-Haim et al., 2007; Mobini and Grant, 2007), an alternative explanation is that the direction of the attentional bias is related to the timing of the effects that are tapped by a specific paradigm, and depends on different underlying cognitive processes. According to the hypervigilance-avoidance hypothesis (Mogg et al., 1997; Mogg and Bradley, 2002), anxious participants may initially orient towards threat, but subsequently direct their attention away in order to reduce their anxiety levels. Although some recent studies provided support for this hypothesis in individuals with SAD (Amir et al., 1998; Mogg et al., 2004), RT data as used in these studies reflect the product of a range of cognitive processes and may therefore be less sensitive for differentiating between biases in early or late stages of information processing. In contrast, event-related potentials (ERPs) recorded from the scalp provide a continuous and high temporal resolution measure of the extent (amplitude) and speed (latency) of cerebral processing, and are therefore particularly suitable for a more refined investigation of the time course of attention allocation to stimuli during emotional processing.

ERPs have been widely used to study processing of emotional material, often including pictures of angry or fearful faces as social threat stimuli. Results of these studies in healthy participants have shown very rapid effects (i.e., $<250$ ms post-stimulus) suggesting early preferential processing of threat-related emotional faces (Eimer and Holmes, 2002; Eger et al., 2003; Ashley et al., 2004; Bar-Haim et al., 2005; Williams et al., 2006), as well as modulation of later stages of threat processing (Eimer and Holmes, 2002; Schupp et al., 2004; Williams et al., 2006). However, studies using ERPs to investigate threat processing in SAD are relatively scarce. In three recent studies Kolassa et al. (Kolassa and Miltner, 2006; Kolassa et al., 2007, 2009) investigated threat processing in patients with SAD using angry compared to neutral and happy faces. Two of these studies (Kolassa et al., 2007, 2009) showed no differential processing of angry faces in SAD during either color or explicit emotion identification of schematic faces. Results of the other study (Kolassa and Miltner, 2006) showed biased early processing of angry photographic faces in patients with SAD, as reflected by enhanced right temporo-parietal N170 amplitudes, during explicit emotion identification but not when emotion processing was implicit (i.e., during gender identification). As suggested by Bar-Haim et al. (2007), biased processing during explicit emotion identification may be contingent on the stimulus being task-relevant, which hinders the generalizability of such findings. Furthermore, both of these studies (Kolassa and Miltner, 2006; Kolassa et al., 2007) focused only on occipito-temporal and parietal electrodes and did not report on the early and late midline positive ERP components that have consistently demonstrated emotional expression effects in healthy participants (see Holmes et al., 2008), and were also shown to be enhanced during implicit angry face processing in high anxious healthy participants (Bar-Haim et al., 2005). Thus, the first aim of the present study was to gain more insight in the temporal dynamics of implicit threat processing in SAD by investigating midline positive ERPs during color-naming in a modified emotional Stroop task with photographic faces. We also included a masked version of this task, to investigate threat processing biases under conditions of restricted awareness.

Our second aim was to investigate the effects of cortisol administration on implicit threat processing in SAD. The stress hormone cortisol plays an important role in the regulation of social motivational processes (e.g., Kalin et al., 1998; van Honk et al., 1998, 2000; Sapolsky et al., 2000; Roelofs et al., 2005, 2009; van Peer et al., 2007), and high cortisol stress-responses have been associated with increased threat avoidance in SAD (Roelofs et al., 2009). Furthermore, cortisol administration has recently been proposed as a possible treatment for SAD, because it reduced self-reported anxiety in social phobic patients during exposure to socioevaluative threat (e.g., Soravia et al., 2006; De Quervain and Margraf, 2008). However, relatively little is known about the effects of cortisol administration on cognitive-emotional processes such as attention to threat. A few recent studies, using explicit emotion evaluation paradigms, showed that cortisol administration can increase angry face processing, especially in high anxious individuals (Putman et al., 2007a; van Peer et al., 2007, 2009). In the present study, we investigate whether cortisol administration has similar effects on the implicit (task-irrelevant) processing of threat.

Implicit threat processing will be measured by recording ERPs during color-identification of angry, happy and neutral faces in both a subliminal (masked) and a supraliminal 
(unmasked) version of a pictorial emotional Stroop task. The tasks are administered in both a placebo and a cortisol administration condition, using a within-subject design. Based on the hypervigilance-avoidance hypothesis (e.g., Mogg et al., 1997) and previous findings of enhanced early threat processing in high anxious participants (e.g., Bar-Haim et al., 2005; Holmes et al., 2008) we expect to find relatively increased early positive midline amplitudes for angry faces in the placebo condition. This effect may be followed by shorter color-naming latencies for angry faces, reflecting threat avoidance (see Putman et al., 2004). Furthermore, we will test whether, and at which stage, this threat processing is affected by cortisol administration.

\section{Methods}

\subsection{Participants}

Eighteen unmedicated patients with SAD participated in the experiment for financial compensation. Group characteristics are presented in Table 1. Patients were recruited at the outpatient departments of three community mental health centers and through advertisements on internet forums. Inclusion criteria were: a primary diagnosis of generalized SAD (according to DSM-IV criteria) and a total score $>60$ at the Liebowitz Social Anxiety Scale (Liebowitz, 1987), righthandedness, normal or corrected-to-normal vision, and age 18-55 years. Exclusion criteria were current diagnosis of major depressive disorder, pregnancy or breast-feeding, clinical significant medical disease, past head injury with loss of consciousness $>5 \mathrm{~min}$, use of psychotropic medication, use of corticosteroids in the 6 months prior to participation, use of cannabis more than once a week or use of any

Table 1 Patient characteristics $(n=17)$.

\begin{tabular}{lll}
\hline Measure & $M$ & SD \\
\hline Age (years) & 31.4 & 10.0 \\
BDI & 12.7 & 6.1 \\
LSAS fear & 43.2 & 7.1 \\
LSAS avoidance & 36.5 & 10.8 \\
LSAS total & 79.7 & 15.9 \\
SPAl social phobia & 132.4 & 20.9 \\
SPAl agoraphobia & 26.4 & 10.0 \\
SPAl total & 106.0 & 21.3 \\
Axis-1 comorbidity & & \\
Comorbid anxiety disorder & & \\
Current mood disorder $^{\mathrm{c}}$ & $n=0$ & \\
\hline
\end{tabular}

Note: (Scale range between parentheses). $\mathrm{BDI}=$ Beck Depression Inventory (0-63); LSAS = Liebowitz Social Anxiety Scale (fear 072, avoidance $0-72$, total $0-144$ ); SPAI = Social Phobia and Anxiety Inventory (social phobia 0-192, agoraphobia 0-78, total $=\mathrm{SP}-\mathrm{Ag})$.

a Assessed using the Structured Clinical Interview for DSM-IV Axis-I Disorders (SCID-I).

b Including panic disorder, agoraphobia, specific phobia, obsessive compulsive disorder, post-traumatic stress disorder and generalized anxiety disorder.

c Including current major depressive episode, mania, hypomania, dysthymic disorder, and bipolar disorder. drugs other than cannabis in the 3 months prior to participation, and use of more than three glasses of alcohol or 20 cigarettes per day. Participants were instructed to minimize physical exercise, not to take large meals, chocolate or caffeine during the morning preceding the experiment, and not to eat, drink low $\mathrm{pH}$ drinks or smoke cigarettes in the hour before the start of the experiment, because these variables can affect saliva cortisol measures. All participants provided written informed consent prior to participation in the study, which was approved by the Medical Ethical Committee of the Leiden University Medical Center. Of the 18 patients tested, one had to be excluded because of missing reaction time data due to technical problems, leaving a total number of 17 participants (7 male, 10 female).

Participants were screened using the Structured Clinical Interview for DSM-IV Axis I Disorders (SCID-I: First et al., 1996) by a trained psychologist at the end of the first testing day to confirm diagnosis for social anxiety disorder and to exclude current major depressive disorder. Participants also completed Dutch versions of the Social Phobia and Anxiety Inventory (SPAl: Turner et al., 1989), and the Beck Depression Inventory (Beck et al., 1979). See Table 1 for questionnaire values.

\subsection{Materials and procedure}

All participants were tested in a cortisol and a placebo condition in a double-blind, within-subject crossover design. The order of cortisol (50 mg hydrocortisone) or placebo (primogel FNA) administration was random and balanced over all participants. The two experimental sessions were 1 week apart. On the days of testing, participants arrived at the laboratory at $1215 \mathrm{~h}$. After a short introduction, drugs were administered orally at $1230 \mathrm{~h}$, followed by a resting period of $1 \mathrm{~h}$ to allow for the cortisol to take effect. During this period, participants completed questionnaires, after which the electrodes for the electrophysiological measurements were placed. The experiment started with a short recording of the resting state electroencephalogram (EEG), followed by a number of additional cognitive tests of which the results will be reported elsewhere (see e.g., van Peer et al., 2009). The emotional Stroop task ( 15 min) was administered at the end of the experiment, approximately $2.5 \mathrm{~h}$ after capsule intake. During task performance, participants sat in an air-conditioned and sound-attenuated room in front of a computer monitor, and the experimenter sat in an adjacent room where the EEG apparatus was located.

\subsection{Cortisol and subjective measures}

Saliva samples were obtained using Salivette collection devices (Sarstedt, Rommelsdorf, Germany). Samples were obtained at four assessment points over a $165 \mathrm{~min}$ period, at respectively $-5 \mathrm{~min}$ (T0), +60 $\mathrm{min}$ (T1), +120 $\mathrm{min}$ (T2), and +160 min (T3) with reference to capsule ingestion. Biochemical analysis of free cortisol in saliva was performed using a competitive electrochemiluminescence immunoassay (ECLIA, Elecsys 2010, Roche Diagnostics), as described elsewhere (van Aken et al., 2003).

Self-reported mood (tension, fatigue, depression, anxiety, and activation) was rated on $100 \mathrm{~mm}$ visual analogue 
scales (VAS) at T0, T1, and T3. In addition, state anxiety (STAl-state: Spielberger, 1983) was measured at T0 and T3.

\subsection{Emotional Stroop task}

Stimuli consisted of photographs of eight actors (four female), each displaying a happy, a neutral and an angry expression (Ekman and Friesen, 1976; Lundqvist et al., 1998). An oval area centered on the face was extracted to remove the hair and non-facial contours. The pictures were equalized in luminance, and colored with a red, green or blue filter. Masking stimuli consisted of oval configurations of randomly cut and reassembled fragments of face stimuli (van Honk et al., 1998). The total stimulus set consisted of 72 target face stimuli ( 8 actors $\times 3$ expressions $\times 3$ colors) and 6 masks ( 2 different $\times 3$ colors). Stimulus presentation and response logging were controlled using $E$-prime software and a serial voice response box and microphone (Psychology Software Tools, inc.). The emotional Stroop task was administered in four phases. Participants started with a practice block of nine trials in which only masks were presented. Next, they completed a masked version of the task of 72 randomized trials. Each trial started with a $750 \mathrm{~ms}$ fixation cross, followed by a very brief $(16.7 \mathrm{~ms}, 2$ frames at $120 \mathrm{~Hz})$ exposure to a target face, which was replaced by a mask of the same color. Participants were instructed to name this color as fast as possible, and vocal response initiation triggered offset of the masks. New trials started after a random inter-trial interval of 2-4 s. The masked version was followed by an unmasked version of the task, which differed only in absence of the masks. Thus, the target stimuli remained visible until registration of responses. To determine whether participants were capable of consciously perceiving the masked facial expressions, the final phase of the task consisted of an awareness check in which a subset of 48 masked faces (each actor and expression twice) was presented to the participants. The instructions explicitly stated that the stimuli consisted of briefly presented faces and participants were asked to indicate (if necessary by guessing) whether the emotional expression of these faces was happy, neutral, or angry by pressing the corresponding response button.

Responses during the masked and unmasked version of the emotional Stroop task were audio-recorded, and incorrect responses (1.3\%) were excluded from the analyses. Reaction times outliers were filtered by using a $<200$ and $>1300 \mathrm{~ms}$ cut-off, and subsequent removal of all RTs exceeding 2.5SD from the individual participants' mean (per task and session). These trials were also excluded from the ERP analyses. Remaining latencies (89.7\% of all trials) were averaged over the facial expression types for each task and condition. Statistical analyses were performed using separate repeated measures ANOVAs for the masked and the unmasked task version, with condition (placebo, cortisol) and stimulus emotion (angry, neutral, happy) as within-subject factors.

\subsection{Electrophysiological recording and analyses}

The electroencephalogram (EEG) was recorded from 19 scalp locations according to the international 10-20 system and referred on-line to $\mathrm{C} 3 / \mathrm{C} 4$. An average earlobe reference was derived off-line. Vertical electro-oculogram (EOG) was recorded bipolarly from the supraorbital and the infraorbital ridge of the right eye, and horizontal EOG from the outer canthi of both eyes. The ground electrode was located at Fpz. EEG impedances were kept below $5 \mathrm{k} \Omega$. The EEG and EOG signals were digitized at $500 \mathrm{~Hz}$ and segmented off-line (using Brain Vision Analyzer software, version 1.05, Brain Products $\mathrm{GmbH}, 1998-2004$ ) into 1000 ms epochs, from 200 ms before to $800 \mathrm{~ms}$ after stimulus onset. Trials with incorrect responses and outlier reaction times were excluded from the analyses. Single trials were corrected for the effects of eye blinks and eye movements using a standard procedure (Gratton et al., 1983). Data were filtered digitally with a $0.1 \mathrm{~Hz}$ high-pass filter ( $24 \mathrm{~dB} /$ oct roll-off) and a $35 \mathrm{~Hz}$ lowpass filter ( $24 \mathrm{~dB} /$ oct). Artifact rejection was performed by removing epochs with activity below $0.50 \mu \mathrm{V}$ for $>100 \mathrm{~ms}$, amplitudes exceeding $\pm 75 \mu \mathrm{V}$, a voltage step per sampling point $>50 \mu \mathrm{V}$, and an absolute difference between two values $>100 \mu \mathrm{V}$. Because of many artifacts in either the F3/F4 or the occipito-temporal (OT) electrodes, artifact rejection and further processing was performed separately for the midline ( $\mathrm{Fz}, \mathrm{Cz}, \mathrm{Pz})$ and the OTelectrodes $(01,02, \mathrm{~T} 5, \mathrm{~T} 6)$ in order to include as many trials as possible in each analysis.

Separate averages were computed for happy, angry and neutral faces as a function of task (masked, unmasked). Six components (P2, N2 and P3 at midline electrodes, and N170, $P 1$, and P2 at OT electrodes) were quantified from the individual participants' waveforms. Peak amplitudes of these components were identified automatically as local maximum relative to the $200 \mathrm{~ms}$ pre-stimulus baseline in defined latency ranges, with manual confirmation. At midline electrodes the P2 (100-250 ms) and N2 (175-300 ms) amplitudes were time-locked to $\mathrm{Cz}$, and $\mathrm{P} 3$ amplitude (275$500 \mathrm{~ms}$ ) was time-locked to Pz (Picton et al., 2000). At OT electrodes N170 amplitude $(110-190 \mathrm{~ms})$ was identified at T5 and T6, and P1 (60-140 ms) and P2 (180-260 ms) amplitudes at 01 and 02 .

The influence of cortisol administration on subjective measures, salivary cortisol, emotional Stroop task performance, and ERP peak amplitudes were tested with repeated measures analyses of variance (ANOVAs) using the Statistical Package for the Social Sciences (SPSS 14.0, SPSS Inc., 19892005). ${ }^{1}$ For the ERP measures, only results involving significant main or interaction effects including Emotion or Condition will be reported. All statistical analyses employed a two-tailed alpha of 0.05 . Effect sizes of significant results are reported as proportion of explained variance (partial eta squared $\left[\eta^{2}\right]$ ). The Greenhouse-Geisser correction was used when appropriate (epsilon $[\varepsilon])$.

\footnotetext{
${ }^{1}$ In addition to the analyses reported below, repeated measures ANOVAs including the factor drug order (cortisol first session or second session) were performed to investigate possible order effects related to repeated administration of the emotional Stroop task. The results of these analyses showed no significant differences between the first and second session in the placebo or the cortisol condition, for either the color-naming latencies or the ERP amplitudes per electrode. Therefore, this factor was not further included in the analyses.
} 
Table 2 Mean free salivary cortisol levels (nmol/l) after placebo and cortisol administration relative to time of capsule intake $(t=0)$.

\begin{tabular}{|c|c|c|c|c|}
\hline \multirow[t]{2}{*}{ Time (min) } & \multicolumn{2}{|c|}{ Placebo } & \multicolumn{2}{|c|}{ Cortisol } \\
\hline & $M$ & $S D$ & $M$ & $S D$ \\
\hline-5 & 9.9 & 3.5 & 9.9 & 3.7 \\
\hline$+60^{* * *}$ & 8.3 & 2.8 & 270.6 & 211.0 \\
\hline$+120^{* \star *}$ & 7.4 & 2.6 & 206.5 & 189.6 \\
\hline$+165^{* \star *}$ & 6.6 & 2.4 & 142.7 & 157.6 \\
\hline
\end{tabular}

\section{Results}

\subsection{Cortisol and subjective measures}

Salivary cortisol (nmol/l) measures (see Table 2) were skewed and therefore log transformed before statistical analysis. The data of one participant were excluded from the cortisol analyses due to unreliable saliva measurements. The results of a $2 \times 4$ repeated measures ANOVA with Condition (placebo, cortisol) and Time (T0, T1, T2, T3) yielded a significant main effect of Condition, $F(1,15)=168.41$, $p=0.000, \eta^{2}=0.92$, and Time, $F(3,45)=45.08, p=0.000$, $\eta^{2}=0.45$, as well as a significant interaction of Condition $\times$ Time, $F(3,45)=63.72, p=0.000, \eta^{2}=0.81$. Followup $F$-tests showed that, as expected, salivary cortisol levels did not differ between conditions before capsule intake (T0), $F(1,15)=0.022, p=0.88)$, but were significantly increased after cortisol administration compared to placebo from $1 \mathrm{~h}$ after capsule intake until the end of the experiment (T1: $F(1,15)=117.53, p=0.000, \eta^{2}=0.89 ; \mathrm{T} 2: F(1,15)=149.87$, $p=0.000, \quad \eta^{2}=0.91 ; \quad$ T3: $\quad F(1,15)=106.46, \quad p=0.000$, $\left.\eta^{2}=0.88\right)$. Note that the emotional Stroop task was administered between T2 and T3 (i.e., between 2 and $2.5 \mathrm{~h}$ after capsule intake).

To investigate effects of cortisol administration on subjective mood (data not shown) we conducted separate repeated measures ANOVAs with Condition (placebo, cortisol) $\times$ Time for STAl-state (T0, T3), and VAS tension, fatigue, depression, anxiety, and activation (T0, T1, T3). Results showed no significant main or interaction effects of Condition on any of the subjective mood measures, except for a trend of Condition on VAS activation $(F(1,16=4.42, p=0.052$, $\eta^{2}=0.21$; all other $F s<1.73$, ps $\left.>0.21, \eta^{2} s<0.10\right)$. Follow-up analyses revealed that reported activation was higher in the placebo compared to the cortisol condition before capsule intake (T0: $F(1,16)=5.33, p=0.035, \eta^{2}=0.25$; placebo: $M=52.8, S D=17.4$; cortisol: $M=40.7, S D=14.2$ ), but not after capsule intake $(\mathrm{T} 1: F(1,16)=2.56, p=0.13$, $\eta^{2}=0.14$; T3: $\left.F(1,16)=0.39, p=0.54, \eta^{2}=0.02\right)$, indicating that this effect was not due to cortisol administration.

\subsection{Behavioral results}

\subsubsection{Awareness check}

A paired samples $t$-test showed that the number of correct responses on the awareness check did not differ as a function of condition (placebo vs. cortisol). We therefore pooled the data
Table 3 Means (and SD) of color-naming latencies (ms).

\begin{tabular}{lll}
\hline & Masked & Unmasked \\
\hline $\begin{array}{lll}\text { Placebo } \\
\text { Angry faces }\end{array}$ & $661(123)$ & $702(134)$ \\
Neutral faces & $657(106)$ & $698(105)$ \\
Happy faces & $670(119)$ & $704(143)$ \\
Cortisol & & \\
Angry faces & $649(131)$ & $669(140)$ \\
Neutral faces & $639(128)$ & $674(144)$ \\
Happy faces & $641(119)$ & $667(138)$ \\
\hline
\end{tabular}

of the two separate measures together to provide a more reliable measure of awareness during emotional Stroop performance (see Putman et al., 2007b). For a binomial test with $n=96$ and $\Pi=0.33$, the individual cut-off score $(p<0.05)$ lies at 41 correct responses. Of the 17 participants, five participants scored 44 or more on the test, and results of the masked task will therefore be reported with and without these participants. The mean number of correct responses of the remaining 12 participants was $33.9(S D=2.9)$.

\subsubsection{Reaction times}

Repeated measures ANOVAs with Condition (placebo, cortisol) and Emotion (angry, happy, neutral) showed no significant effects on color-naming latencies in either the masked task version, all $F s<1.25$, ps $>0.28$ (without the five participants that scored above chance level at the awareness check, all $F \mathrm{~s}<1.9$, ps $>0.19$ ), or the unmasked task version, all $F \mathrm{~s}<3.16$, ps $>0.090$. See Table 3 .

\subsection{ERP results masked task: midline electrodes (Fz, Cz, Pz)}

See Figure 1 for grand average ERPs at $\mathrm{Fz}, \mathrm{Cz}$, and Pz in the masked task.

\subsubsection{P2 amplitude}

The repeated measures ANOVA with Condition (placebo, cortisol), Emotion (angry, happy, neutral) and Electrode (Fz, Cz, $\mathrm{Pz}$ ) showed a significant interaction of Condition $\times$ Emotion on the $\mathrm{P} 2$ amplitudes in the masked task version, $F(2,32)=3.99$, $p=0.028, \eta^{2}=0.20$. Follow-up testing to clarify the nature of this interaction revealed that the effect of Emotion was significant in the placebo condition, $F(2,32)=4.81, p=0.015$, $\eta^{2}=0.23$, but not in the cortisol condition, $F(2,32)=0.68$, $p=0.52$. In the placebo condition, the $\mathrm{P} 2$ amplitudes were significantly larger (more positive) for angry faces compared to both neutral, $F(1,16)=7.93, p=0.012, \eta^{2}=0.33$, and happy faces $F(1,16)=4.60, p=0.048, \eta^{2}=0.22$, but did not differ significantly between happy and neutral faces, $F(1,16)=0.59$, $p=0.46$. Cortisol administration tended to decrease the $\mathrm{P} 2$ amplitudes compared to placebo for angry faces, $F(1,16)=3.88, p=0.066, \eta^{2}=0.20$, but did not significantly affect the P2 amplitudes for neutral, $F(1,16)=1.91, p=0.19$, or happy faces, $F(1,16)=0.15, p=0.71$. No other effects reached significance, all $F s<2.2$, ps $>0.12$. The Condition $\times$ Emotion interaction remained significant when the participants that scored above chance level on the awareness check $(n=5)$ were excluded from the analyses, $F(2,22)=3.51$, 
(a) Masked placebo
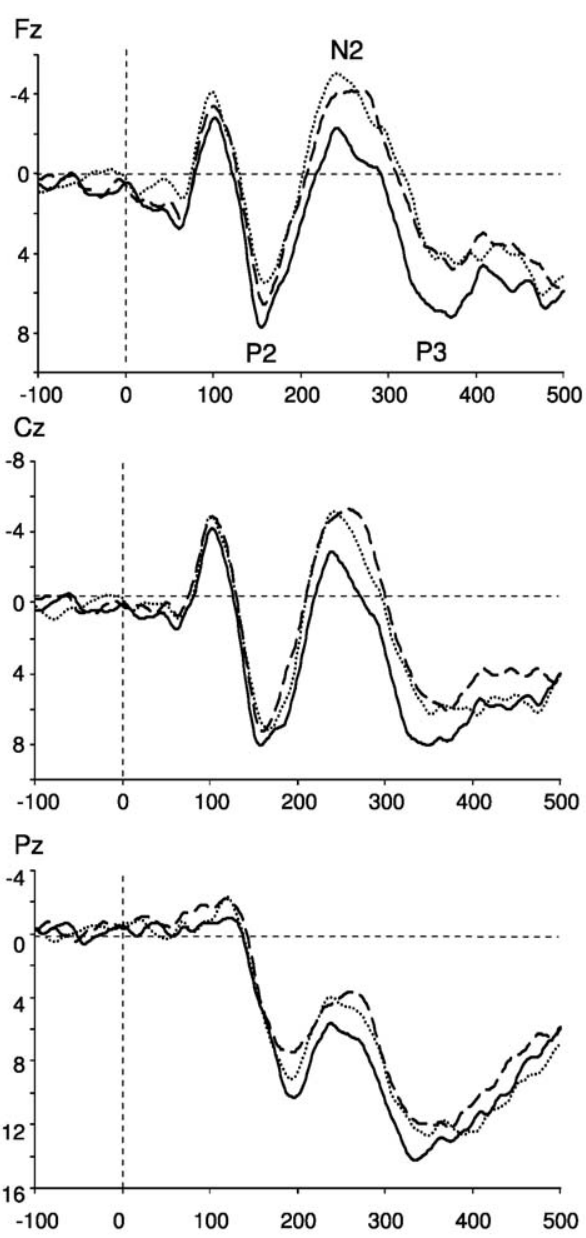

(b) Masked cortisol
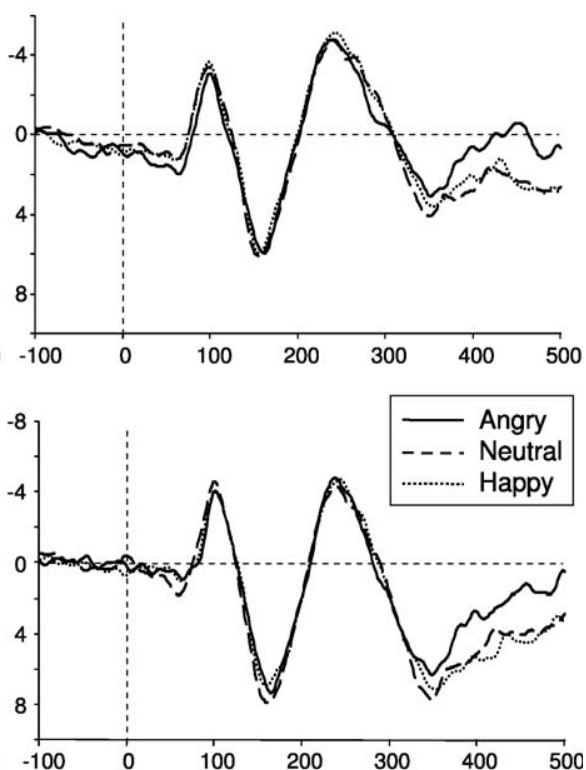

$[\mu \mathrm{V}]$

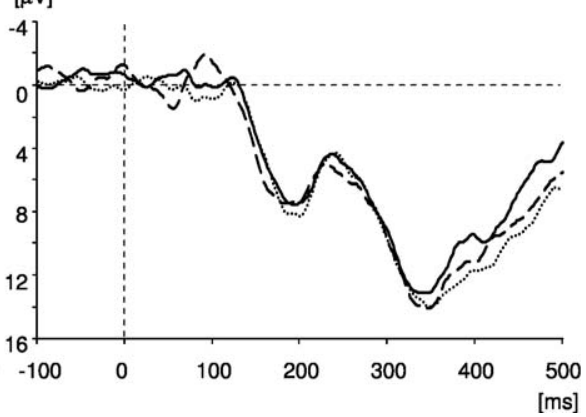

Figure 1 Stimulus synchronized grand average ERP waveforms at midline electrodes ( $\mathrm{Fz}, \mathrm{Cz}, \mathrm{Pz})$ in the masked emotional Stroop task after placebo (left) and cortisol (right) administration. Results showed a significant Condition $\times$ Emotion interaction at P2 and N2 components, reflecting significantly more positive amplitudes for angry faces in the placebo condition. This effect disappeared after cortisol administration.

$p=0.048, \eta^{2}=0.24$. The P2 amplitudes for angry faces in the placebo condition remained significantly more positive compared to happy, $F(1,11)=5.42, p=0.040, \eta^{2}=0.33$, and neutral faces, $F(1,11)=5.11, p=0.045, \eta^{2}=0.32$. In addition, the decrease in P2 amplitudes after cortisol administration compared to placebo for angry faces was now significant, $F(1,11)=7.31, p=0.021, \eta^{2}=0.40$.

\subsubsection{N2 amplitude}

The results of the $\mathrm{N} 2$ amplitudes in the masked task version showed a significant main effect of Emotion, $F(2,32)=3.81$, $p=0.048, \varepsilon=0.74, \eta^{2}=0.19$, which was further qualified by $\mathrm{a}$ significant Condition $\times$ Emotion interaction, $F(2,32)=3.97$, $p=0.029, \eta^{2}=0.20$. In line with the results of the P2 amplitudes, the effect of Emotion was significant in the placebo condition, $F(2,32)=6.92, p=0.003, \eta^{2}=0.30$, but not after cortisol administration, $F(2,32)=0.34, p=0.71$. In the placebo condition, the N2 amplitudes for angry faces were significantly decreased (i.e., more positive) compared to both neutral, $F(1,16)=8.85, p=0.009, \eta^{2}=0.36$, and happy faces, $F(1,16)=10.16, p=0.006, \eta^{2}=0.39$. The difference in N2 amplitude for neutral compared to happy faces was not significant, $F(1,16)=0.03, p=0.87$. Cortisol administration resulted in significantly more negative N2 amplitudes compared to placebo for angry faces, $F(1,16)=14.38, p=0.002$, $\eta^{2}=0.47$, but did not affect the N2 amplitudes for neutral, $F(1,16)=0.14, p=0.71)$ or happy faces, $F(1,16)=0.17$, $p=0.69$. No other effects including Emotion or Condition reached significance, all $F s<3.1$, ps $>0.07$. The Condition $\times$ Emotion interaction remained significant when the participants that scored above chance level on the awareness check $(n=5)$ were excluded from the analyses, $F(2,22)=5.12, p=0.015, \eta^{2}=0.32$. The N2 amplitudes for angry faces in the placebo condition remained significantly decreased (i.e., more positive) compared to neutral, $F(1,11)=9.22, \quad p=0.011, \quad \eta^{2}=0.46$, and happy faces, $F(1,11)=10.08, p=0.009, \eta^{2}=0.48$, and the effect of cortisol administration on the $\mathrm{N} 2$ amplitudes for angry faces also remained significant, $F(1,11)=15.67, p=0.002, \eta^{2}=0.59$.

\subsubsection{P3 amplitude}

In contrast to the P2 and N2 amplitudes, the P3 amplitudes did not show significant effects for Emotion, $F(2,32)=0.01$, $p=0.99$, or Condition $\times$ Emotion, $F(2,32)=2.34, p=0.11$. 
Table 4 Means (and standard errors) of peak amplitudes $(\mu \mathrm{V})$ at midline and occipito-temporal electrodes as a function of condition, task and stimulus emotion.

\begin{tabular}{|c|c|c|c|c|c|c|c|}
\hline & & $\begin{array}{l}\text { Masked } \\
\text { Angry }\end{array}$ & Neutral & Happy & $\begin{array}{l}\text { Unmasked } \\
\text { Angry }\end{array}$ & Neutral & Happy \\
\hline \multicolumn{8}{|c|}{ Midline } \\
\hline \multirow[t]{2}{*}{ P2 } & Placebo & $10.1(1.4)$ & $8.0(1.4)$ & $8.4(1.3)$ & $14.1(1.1)$ & $11.3(12.3)$ & $12.3(1.3)$ \\
\hline & Cortisol & $8.2(1.2)$ & $9.0(1.3)$ & $8.8(1.3)$ & $13.2(1.2)$ & $12.5(1.4)$ & $12.5(1.4)$ \\
\hline \multirow[t]{2}{*}{ N2 } & Placebo & $-0.9(1.3)$ & $-3.5(1.3)$ & $-3.4(1.5)$ & $2.7(1.5)$ & $0.8(1.6)$ & $0.1(1.7)$ \\
\hline & Cortisol & $-3.6(1.3)$ & $-3.2(1.2)$ & $-3.8(1.2)$ & $1.2(1.5)$ & $0.1(1.4)$ & $0.8(1.4)$ \\
\hline \multirow[t]{2}{*}{ P3 } & Placebo & $11.8(1.6)$ & $10.5(1.5)$ & $10.7(1.3)$ & $14.2(1.8)$ & $12.5(1.4)$ & $14.3(1.6)$ \\
\hline & Cortisol & $8.9(1.0)$ & $10.3(1.4)$ & $10.2(1.3)$ & $12.0(1.4)$ & $11.6(1.5)$ & $12.0(1.4)$ \\
\hline \multicolumn{8}{|c|}{ Occipito-temporal } \\
\hline \multirow[t]{2}{*}{ N170 } & Placebo & $-3.0(1.0)$ & $-4.0(1.0)$ & $-3.5(0.9)$ & $-8.5(1.6)$ & $-8.6(1.1)$ & $-9.1(1.4)$ \\
\hline & Cortisol & $-3.3(1.0)$ & $-2.9(1.1)$ & $-3.6(1.0)$ & $-8.1(1.3)$ & $-7.7(1.2)$ & $-8.2(1.2)$ \\
\hline \multirow[t]{2}{*}{ P1 } & Placebo & $7.4(1.7)$ & $7.0(1.7)$ & $7.7(1.8)$ & $6.4(1.5)$ & $6.4(1.7)$ & $6.9(1.6)$ \\
\hline & Cortisol & $8.1(1.9)$ & $8.1(1.6)$ & $8.9(1.7)$ & 6.7 (1.5) & $7.5(1.8)$ & $7.4(1.7)$ \\
\hline \multirow[t]{2}{*}{ P2 } & Placebo & $16.6(2.1)$ & $15.7(1.8)$ & $16.0(1.9)$ & $13.5(1.7)$ & $14.0(1.7)$ & $13.3(1.7)$ \\
\hline & Cortisol & $16.5(1.8)$ & $17.2(1.9)$ & $17.3(1.7)$ & $13.4(1.6)$ & $14.0(1.7)$ & $12.8(1.6)$ \\
\hline
\end{tabular}

Note: Midline at Fz/Cz/Pz electrodes; occipito-temporal: N170 at T5/T6 electrodes; P1 and P2 at 01/02 electrodes.

Furthermore, no other effects including Emotion or Condition reached significance, all $F \mathrm{~s}<3.8, \mathrm{ps}>0.05$.

\subsection{Occipito-temporal electrodes (T5, T6, 01, 02)}

The repeated measures ANOVAs showed no significant results for the N170 amplitudes (T5, T6), all Fs $<3.2$, ps $>0.05$, or the occipital P2 amplitudes $(01,02)$, all $F \mathrm{~s}<2.3, \mathrm{ps}>0.12$, in the masked task version. For the occipital P1 amplitudes results showed a significant Emotion $\times$ Electrode interaction, $F(2,32)=6.89, p=0.003, \eta^{2}=0.30$, reflecting a significant effect of Emotion at 02, $F(2,32)=3.89, p=0.031, \eta^{2}=0.20$, but this effect disappeared after the participants that scored above chance level at the awareness check were excluded from the analyses, $F(2,22)=2.32, p=0.12$. No other effects reached significance, all $F s<3.7, p s>0.07$. See Table 4 .

\subsection{ERP results unmasked task: midline electrodes ( $\mathrm{Fz}, \mathrm{Cz}, \mathrm{Pz})$}

See Table 4 for mean amplitudes at midline and occipitotemporal electrodes in the unmasked task.

\subsubsection{P2 amplitude}

In contrast to the results of the masked task, the interaction of Condition $\times$ Emotion on the midline $P 2$ amplitudes was not significant in the unmasked task, $F(2,32)=1.85, p=0.17$. We did find a significant main effect of Emotion, $F(2,32)=5.32$, $p=0.010, \eta^{2}=0.25$. Follow-up $F$-tests showed that the P2 amplitudes were significantly increased for angry compared to neutral faces, $F(1,16)=12.94, p=0.002, \eta^{2}=0.45$, and showed a tendency in the same direction for angry compared to happy faces, $F(1,16)=3.95, p=0.064, \eta^{2}=0.20$. The difference between the $\mathrm{P} 2$ amplitudes for neutral and happy faces was not significant, $F(1,16)=0.95, p=0.34$. No other effects including Emotion or Condition reached significance, all $F s<1.2$, ps $>0.34$.

\subsubsection{N2 amplitude}

Results of the N2 amplitudes showed a significant Electrode $\times$ Emotion interaction, $F(4,64)=7.09, p=0.001, \varepsilon=0.62$, $\eta^{2}=0.31$. Follow-up $F$-tests showed a significant effect of Emotion only at Pz, $F(2,32)=6.05, p=0.006, \eta^{2}=0.27$, reflecting significantly less negative amplitudes for angry compared to happy faces, $F(1,16)=11.24, p=0.004, \eta^{2}=0.41$, and a trend in the same direction for angry compared to neutral faces, $F(1,16)=3.63, p=0.075, \eta^{2}=0.27$. This result is in line with the findings for the masked task. No other effects including Emotion or Condition reached significance, all $F \mathrm{~s}<3.2$, ps $>0.05$.

\subsubsection{P3 amplitude}

In line with the findings of the masked task, the results for the unmasked task version showed no significant effects involving Emotion or Condition on the P3 amplitudes, all Fs $<1.9$, ps $>0.18$.

\subsection{Occipito-temporal electrodes (T5, T6, 01, 02)}

In line with the masked task, results of the unmasked task version showed no significant effects on the N170 amplitudes (T5, T6), all $F s<4.4$, ps $>0.05$, or the occipital P2 amplitudes $(01,02), F s<2.2$, ps $>0.16$. The occipital P1 amplitudes did not show any significant results in the unmasked task either, all $F \mathrm{~s}<2.25$, ps $>12$.

\subsection{Relationship between threat processing and social anxiety}

Since previous studies have shown significant relationships between ERP amplitudes during threat processing and severity of (social) anxiety (see Bar-Haim et al., 2005; Kolassa and Miltner, 2006), including effects of cortisol administration (van Peer et al., 2009) we explored whether any of the significant ERP effects involving angry faces in the present study were influenced by individual differences in social 


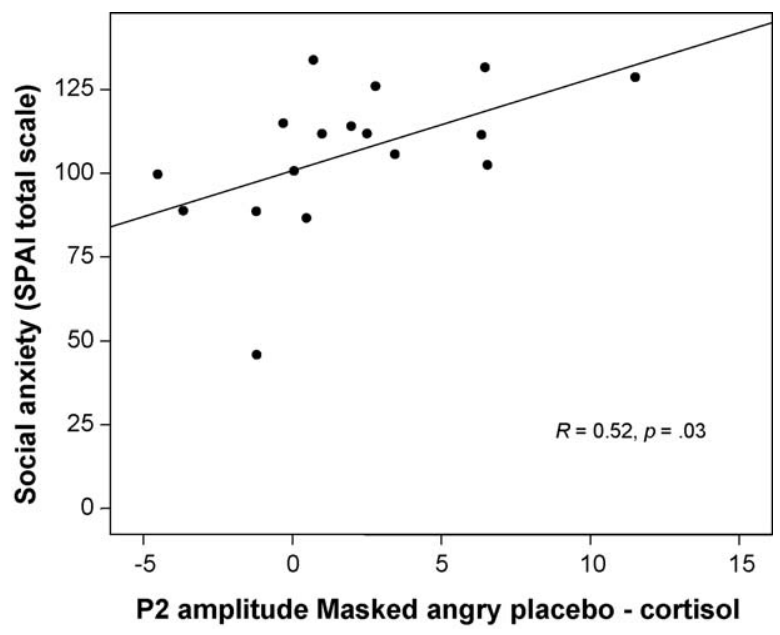

Figure 2 Correlation between social anxiety (SPAI total score) and cortisol-induced change in P2 amplitude for angry faces in the masked emotional Stroop task (i.e., P2 masked angry placebo - cortisol). Positive values on the $x$-axis indicate larger P2 amplitudes on midline electrodes ( $\mathrm{Fz}, \mathrm{Cz}, \mathrm{Pz})$ in the placebo condition, compared to the cortisol condition. The scatterplot shows that high levels of social anxiety are associated with a larger cortisol-induced decrease in P2 amplitudes for masked angry faces, indicating a decrease in threat processing (after cortisol administration) under conditions of restricted awareness. Note: The correlation was somewhat depressed by one participant (see the lower left corner of the graph). Without this participant the correlation was $R=0.56, p=0.024$, but this person was no statistical outlier (Mahalanobis $D^{2}=8.64, p=0.02$ ).

anxiety. We investigated this by including social anxiety as a continuous factor in the repeated measures analyses (ANCOVA: see Judd et al., 2001) for the ERP amplitudes that showed a significant effect for angry faces. Significant interactions with social anxiety were followed up by calculating Pearson correlations between social anxiety and the difference score of the relevant within-subjects factor.

Results showed that the effect of Condition on the P2 amplitudes for angry faces in the masked task was significantly influenced by individual differences in social anxiety, as reflected by a significant interaction of Condition with the SPAI total score, $F(1,15)=5.63, p=0.031, \eta^{2}=0.27$. Pearson correlations between total scores on the SPAI and the cortisolinduced change in P2 amplitude for masked angry faces (i.e., P2 amplitude masked angry placebo - cortisol) showed that the direction of this correlation was positive $(R=0.52, p=0.031)$, indicating that patients with higher levels of social anxiety showed a significantly stronger decrease in P2 amplitudes after cortisol administration compared to placebo (see Figure 2). This effect was not significant for the SPAI social phobia scores, although the correlation showed the same direction of effects $(R=0.33, p=0.20)$. None of the other ERPamplitude effects for angry faces in this study were significantly associated with individual differences in social anxiety.

\section{Discussion}

The major aims of the present study were to gain more insight in the temporal dynamics of biased processing of implicit social threat in SAD, and to explore the effect of cortisol administration on such processing. This was investigated by measuring ERPs during color-naming of masked and unmasked emotional faces in a modified emotional Stroop task after placebo and cortisol administration in participants with a clinical diagnosis of generalized SAD.

\subsection{Early processing advantage for angry faces in SAD}

First, the ERP results showed an early processing bias for social threat stimuli in the placebo condition, as reflected by increased (more positive) P2 amplitudes for angry compared to neutral and happy faces, even when they were presented under conditions of restricted awareness. ERP amplitudes for angry faces continued to be significantly more positive in the time-window of the N2 component on all electrodes in the masked task, as well as on Pz electrode in the unmasked task. Increased amplitudes of early positive frontocentral ERP components in reaction to threat-related (i.e., angry or fearful) emotional faces have previously been reported in studies in healthy participants, and are generally interpreted as reflecting increased allocation of processing resources to motivationally significant stimuli (Eimer and Holmes, 2002; Eimer et al., 2003; Bar-Haim et al., 2005; Williams et al., 2006).

To our knowledge, the present study is the first showing increased early processing of implicit social threat reflected by ERPs in participants with a clinical diagnosis of SAD. This finding is in line with a range of behavioral studies showing hypervigilance to social threat in SAD (see Bar-Haim et al., 2007; Mobini and Grant, 2007 for recent reviews), and provides support for the notion that such vigilance occurs in early stages of information processing (e.g., Mogg et al., 1997; Amir et al., 1998; Mogg and Bradley, 2002). ERP studies investigating angry face processing in relation to (social) anxiety are scarce, but there are some recent findings in non-clinical samples providing support for increased early threat processing in (socially) anxious participants. In line with our results, Bar-Haim et al. (2005) found enhanced P2 amplitudes to angry faces in high compared to low trait anxious healthy participants, indicating that implicit early threat processing can be modulated by anxiety (cf. Holmes et al., 2008; Moser et al., 2008). In addition, Rossignol et al. (2007) reported facilitated detection of subtle changes in anger expression during face repetition in participants with non-clinical social anxiety compared to low socially anxious participants, as reflected by a reduced N2b wave around 300 ms post-stimulus. Only a few previous ERP studies investigated angry face processing in participants with clinically diagnosed SAD (Kolassa and Miltner, 2006; Kolassa et al., 2007, 2009). In contrast to the present study, these found no evidence for increased early processing of task-irrelevant angry faces, although enhanced right temporo-parietal N170 amplitudes were found during explicit emotion identification of angry faces in one study (Kolassa and Miltner, 2006). The lack of a processing bias for implicit social threat in these studies may be partly due to the use of schematic face stimuli (Kolassa et al., 2007, 2009) which show a different electrophysiological response pattern than photographic faces (Kolassa et al., 2007) and may be less sensitive for detecting 
differential social threat processing in SAD. Furthermore, all of these studies (Kolassa and Miltner, 2006; Kolassa et al., 2007, 2009) focused on occipito-temporal electrodes and did not report on the early and late midline positive ERP components that we investigated in the present study, and which have consistently demonstrated emotional expression effects in healthy participants (see Holmes et al., 2008). Emotional modulation of the $\mathrm{N} 170$ component has been less consistently found and may be only present when the identification of the facial emotion is explicitly task-relevant, as suggested by Kolassa et al. (2009, see also Kolassa and Miltner, 2006), which would explain why we did not observe this effect in the present study.

\subsection{Effects of cortisol on implicit threat processing}

Our second main finding was that the early threat bias, reflected by increased P2 amplitudes for angry faces, significantly decreased (and disappeared) after cortisol administration in the masked task. Moreover, this effect was stronger for participants with higher levels of social anxiety. Both the finding that cortisol administration only significantly affected P2 amplitudes for angry faces, and the finding that the magnitude of this effect was related to severity of social anxiety are consistent with the results of van Peer et al. (2009). However, the direction of the effect of cortisol administration in the present study was opposite to the findings of this previous study. van Peer et al. (2009) investigated the effect of cortisol administration on ERPs in patients with SAD using a RT paradigm measuring social approach and avoidance behavior in reaction to happy and angry faces. In contrast to the decrease in P2 amplitudes for masked angry faces in the present study, the results of van Peer et al. (2009) showed cortisol-induced increases in these same amplitudes, indicating enhanced processing, during avoidant responses to angry faces in patients with high levels of social anxiety (see also van Peer et al., 2007). Notably, both of these tasks (i.e., the approach-avoidance task of van Peer et al. (2009) and the emotional Stroop task described in the present article) were administered during the same experiment, and thus concerned the same group of participants. Therefore, the contrasting findings are most likely explained by task-related differences. First, cortisol only significantly decreased P2 amplitudes for masked angry faces in the present study, whereas stimuli in the previous study were all unmasked. Several authors have suggested that processing of unmasked threat stimuli may be affected by mood-controlling strategies in high socially anxious individuals (e.g., Williams et al., 1996; Mogg and Bradley, 2002), whereas such strategies are minimized during masked presentation. Consistent with this notion, behavioral evidence for modulation of angry face processing by social anxiety has been previously found in masked, but not unmasked facial emotional Stroop tasks (Putman et al., 2004). Similarly, angry face processing was significantly related to baseline endogenous cortisol levels in masked, but not unmasked versions of this task (van Honk et al., 1998). Thus, masking has an important influence on the processing of these stimuli, and the preclusion of cognitive control processes could provide grounds for an interaction with cortisol.
A second important factor is that van Peer et al. (2009) used an explicit affect-evaluation task, whereas the emotional expression of the faces in the present task was implicit and task-irrelevant. Several studies suggest that task-relevance vs. irrelevance of emotional expression can have a significant effect on ERPs related to early threat processing (see e.g., Bar-Haim et al., 2005; Kolassa and Miltner, 2006; Eimer and Holmes, 2007; Kolassa et al., 2009). Furthermore, effects of cortisol on processing of negative or threatening emotional information may differ depending on task-relevance of these emotional stimuli. To our knowledge, only two previous studies investigated effects of cortisol administration on processing of task-irrelevant (or distracting) emotional stimuli. Putman et al. (2007b) reported increased color-naming latencies (reflecting interference or threat vigilance) for masked fearful compared to neutral faces on an emotional Stroop task after placebo administration. This effect was abolished after cortisol administration. Interestingly, this cortisol-induced decrease in fear processing was most pronounced in participants with high self-reported trait anxiety. More recently, Oei et al. (2009) found reduced interference by task-irrelevant negative pictures in a modified Sternberg working memory task after cortisol administration compared to placebo. Although both of these studies were conducted in healthy young men and did not use angry face stimuli, the pattern of results (reduced processing of task-irrelevant negative stimuli) is consistent with the present findings. In contrast, the findings of van Peer et al. (2009) are in line with other studies showing increased angry face processing after cortisol administration in explicit emotion evaluation paradigms (Putman et al., 2007a; van Peer et al., 2007). Thus, task or goal-relevance may be an important factor modulating the effects of corticosteroids on information processing, resulting in cortisol-induced increases in threat processing and avoidance when the stimuli are task-relevant, and inhibition of threat processing when the emotional stimuli are task irrelevant or distracting. These findings are consistent with the view that cortisol generally facilitates processing and adaptive behavior that is most relevant to the situation, as advocated by De Kloet et al. (1999) based on animal studies. Further research is needed to directly and systematically investigate the role of such task-related factors on the effect of cortisol on early attention processes.

The results of the present study suggest that social threat stimuli automatically attract more attention in patients with SAD at very early stages of information processing, and that cortisol administration decreases this threat bias under conditions of restricted awareness. There are, however, some limitations that should be discussed.

First, we did not find any significant behavioral results, although this is in line with the results of other studies in patients with SAD using a similar paradigm (Kolassa and Miltner, 2006). van Hooff et al. (2008) suggested that a lack of behavioral findings in emotional Stroop studies with ERP could be due to the use of relatively long inter-trial intervals (ITIs, 2-4 s in the present study). However, previous behavioral studies using the same paradigm (with long ITIs) as the present study have shown it to be sensitive to detect attentional biases in high socially anxious healthy participants (see Putman et al., 2004), which makes this explanation less likely. A second possibility, as described in the introduction, 
is that behavioral results of the emotional Stroop task are less sensitive to detect attentional biases in social phobic patients, because RT data reflect the combined product of a range of cognitive processes, including possibly opposite biases in early and late stages of information processing. For this reason we included measurement of ERPs during task performance. Nevertheless, the demonstration of an attentional bias for threat using RTs would be helpful as a confirmatory measure to strengthen conclusions regarding ERP effects (e.g., Holmes et al., 2009). Inclusion of a larger subject sample is also recommended to increase the statistical power to detect small or medium sized effects.

A second limitation is that we did not include a nonanxious control group, and therefore cannot conclude whether our finding of an early threat bias, or the effects of cortisol on this bias, are specific to social anxiety. The finding that effects of cortisol on early threat processing were stronger in patients with higher levels of social anxiety does provide tentative support for an increased sensitivity in high anxious patients, although this is limited by the fact that the participants are all within a restricted diagnostic range. In addition, a recent study by Putman et al. (2007b) using a highly similar Emotional Stroop task, showed a cortisolinduced decrease in fear processing that was most pronounced in healthy participants with high self-reported levels of trait anxiety. This finding supports the notion that anxious participants may show increased sensitivity to cortisol effects on threat processing compared to non-anxious participants (see also Roelofs et al., 2009; van Peer et al., 2007). Nevertheless, future ERP research including a matched healthy control group is necessary to conclude whether the effects of cortisol on implicit threat processing as found in the present study are specific to (or increased in) socially anxious participants, or reflect a process that can be found in the general population.

Third, the masked task was administered prior to the unmasked task in all participants in order to minimize the chance that participants would consciously perceive the masked facial expressions due to, for example, priming effects. Although this does not affect our main findings, it may have confounded effects of masking with effects of repeated administration and should be accounted for in future studies.

Finally, in the present study we administered cortisol to investigate its causal influence on cognitive-emotional processes that play an important role in social anxiety disorder. Although exogenous administration studies are better suited to investigate the causal role of cortisol compared to e.g., stress induction, as they constrain effects related to arousal and noradrenergic activation, it should be noted that the results of these studies cannot simply be generalized to naturalistic situations with elevated cortisol levels. Thus, further research is needed to assess the ecological validity of our findings by comparing them with the effects of endogenous cortisol increases. In addition, the effects of cortisol in the present study relied on a single high $(50 \mathrm{mg})$ dose, whereas dose-response studies in the field of memory research have shown an inverted $U$-shape relationship between cognition and glucocorticoid (GC) levels. That is, very high and low GC doses caused memory impairment, whereas moderate doses caused memory enhancement (see e.g., Lupien et al., 1999). Future studies including more moderate doses of cortisol are needed to investigate whether different doses of cortisol result in a similar inverted U-shape effect on early threat processing.

To conclude, this study provided the first psychophysiological evidence for increased early processing of implicit social threat in participants with a clinical diagnosis of SAD, and showed that cortisol administration decreased this threat bias under conditions of restricted awareness. Together with previous findings (van Peer et al., 2009) these results indicate a highly specific effect of cortisol on early motivated attention to social threat, and highlight the importance of motivational context (goal-relevance) and symptom severity.

\section{Role of funding source}

The work of K. Roelofs was supported by a VIDI Grant (\#45207-008) from the Netherlands Organization for Scientific Research (NWO). NWO had no further role in study design; in the collection, analysis and interpretation of data; in the writing of the report; and in the decision to submit the paper for publication.

\section{Conflict of interest}

None declared.

\section{Acknowledgements}

The authors thank Jack van Honk and Erno Hermans for the task software; Miranda van den Berge, Laila Benbrahim, Liesbeth van Beemen, Janne Hofmeester, and the therapists from the anxiety unit of PsyQ, The Hague and Rivierduinen, Leiden, for their assistance in patient recruitment; the lab assistants of the Department of Clinical Neurophysiology of the Leiden University Medical Centre (LUMC) for their assistance in data collection; Cor Kramer and Robert Reijntjes for the technical support; and Hans van Pelt and Jolanda Verhagen for the cortisol analyses at the Clinical Chemical lab of the LUMC.

\section{References}

Amir, N., Foa, E.B., Coles, M.E., 1998. Automatic activation and strategic avoidance of threat-relevant information in social phobia. J. Abnorm. Psychol. 107, 285-290.

Amir, N., Freshman, M., Foa, E., 2002. Enhanced Stroop interference for threat in social phobia. J. Anxiety Disord. 16, 1-9.

Ashley, V., Vuilleumier, P., Swick, D., 2004. Time course and specificity of event-related potentials to emotional expressions. Neuroreport 15, 211-216.

Asmundson, G.J.G., Stein, M.B., 1994. Selective processing of social threat in patients with generalized social phobia-evaluation using a dot-probe paradigm. J. Anxiety Disord. 8, 107-117.

Bar-Haim, Y., Lamy, D., Glickman, S., 2005. Attentional bias in anxiety: a behavioral and ERP study. Brain Cogn. 59, 11-22.

Bar-Haim, Y., Lamy, D., Pergamin, L., Bakermans-Kranenburg, M.J., van IJzendoorn, M.H., 2007. Threat-related attentional bias in anxious and nonanxious individuals: a meta-analytic study. Psychol. Bull. 133, 1-24.

Beck, A.T., Rush, A.J., Hollon, S.D., Emery, G., 1979. Cognitive therapy of depression. Wiley, New York.

Chen, Y.P., Ehlers, A., Clark, D.M., Mansell, W., 2002. Patients with generalized social phobia direct their attention away from faces. Behav. Res. Ther. 40, 677-687. 
Clark, D.M., Wells, A., 1995. A cognitive model of social phobia. In: Heimberg, R.G., Liebowitz, M., Hope, D.A., Schneier, F.R. (Eds.), Social Phobia: Diagnosis, Assessment and Treatment. Guilford Press, New York, pp. 69-93.

Condren, R.M., O’Neill, A., Ryan, M.C.M., Barrett, P., Thakore, J.H., 2002. HPA axis response to a psychological stressor in generalised social phobia. Psychoneuroendocrinology 27, 693-703.

De Kloet, E.R., Oitzl, M.S., Joëls, M., 1999. Stress and cognition: are corticosteroids good or bad guys? Trends Neurosci. 22, 422426.

De Quervain, D.J.F., Margraf, J., 2008. Glucocorticoids for the treatment of post-traumatic stress disorder and phobias: a novel therapeutic approach. Eur. J. Pharmacol. 583, 365-371.

Eger, E., Jedynak, A., Iwaki, T., Skrandies, W., 2003. Rapid extraction of emotional expression: evidence from evoked potential fields during brief presentation of face stimuli. Neuropsychologia 41, 808-817.

Eimer, M., Holmes, A., 2002. An ERP study on the time course of emotional face processing. Neuroreport 13, 427-431.

Eimer, M., Holmes, A., McGlone, F.P., 2003. The role of spatial attention in the processing of facial expression: an ERP study of rapid brain responses to six basic emotions. Cogn. Affect. Behav. Neurosci. 3, 97-110.

Eimer, M., Holmes, A., 2007. Event-related brain potential correlates of emotional face processing. Neuropsychologia 45, 15-31.

Ekman, P., Friesen, W.V., 1976. Pictures of Facial Affect. Consulting Psychologist Press, Palo Alto, CA.

First, M.B., Spitzer, R.L., Gibbon, M., Williams, J.B., 1996. Structured Clinical Interview for the DSM-IV Axis I Disorders Version 2. Biometrics Research, New York.

Gilboa-Schechtman, E., Foa, E.B., Amir, N., 1999. Attentional biases for facial expressions in social phobia: the face-in-the-crowd paradigm. Cogn. Emot. 13, 305-318.

Gratton, G., Coles, M.G.H., Donchin, E., 1983. A new method for offline removal of ocular artifact. Electroencephal. Clin. Neurophysiol. 55, 468-484.

Hermans, E.J., van Honk, J., 2006. Toward a framework for defective emotion processing in social phobia. Cogn. Neuropsychiatry 11, 307-331.

Holmes, A., Nielsen, M.K., Green, S., 2008. Effects of anxiety on the processing of fearful and happy faces: an event-related potential study. Biol. Psychol. 77, 159-173.

Holmes, A., Bradley, B.P., Kragh Nielsen, M., Mogg, K., 2009. Attentional selectivity for emotional faces: evidence from human electrophysiology. Psychophysiology 46, 62-68.

Judd, C.M., Kenny, D.A., McClelland, G.H., 2001. Estimating and testing mediation and moderation in within-subject designs. Psychol. Methods 6, 115-134.

Kalin, N.H., Larson, C., Shelton, S.E., Davidson, R.J., 1998. Asymmetric frontal brain activity, cortisol, and behavior associated with fearful temperament in rhesus monkeys. Behav. Neurosci. 112, 286-292.

Kolassa, I.T., Miltner, W.H.R., 2006. Psychophysiological correlates of face processing in social phobia. Brain Res. 1118, 130-141.

Kolassa, I.T., Kolassa, S., Musial, F., Miltner, W.H.R., 2007. Eventrelated potentials to schematic faces in social phobia. Cogn. Emot. 21, 1721-1744.

Kolassa, I.T., Kolassa, S., Bergmann, S., Lauche, R., Dilger, S., Miltner, W.H.R., Musial, F., 2009. Interpretive bias in social phobia: an ERP study with morphed emotional schematic faces. Cogn. Emot. 23, 69-95.

Liebowitz, M.R., 1987. Social phobia. In: Ban, T.A., Pichot, P., Poldinger, W. (Eds.), Modern Problems of Pharmacopsychiatry. 22nd ed. Karger, Basel, pp. 141-173.

Lundqvist, D., Flykt, A., Öhman, A., 1998. The Karolinska Directed Emotional Faces. Karolinska Institute, Stockholm.

Lupien, S.J., Gillin, C.J., Hauger, R.L., 1999. Working memory is more sensitive than declarative memory to the acute effects of corticosteroids: a dose-response study in humans. Behav. Neurosci. $113,420-430$

Mansell, W., Clark, D.M., Ehlers, A., Chen, Y.P., 1999. Social anxiety and attention away from emotional faces. Cogn. Emot. 13, 673690.

Mattia, J.I., Heimberg, R.G., Hope, D.A., 1993. The revised Stroop color-naming task in social phobics. Behav. Res. Ther. 31, 305313.

Mobini, S., Grant, A., 2007. Clinical implications of attentional bras in anxiety disorders: an integrative literature review. Psychotherapy $44,450-462$.

Mogg, K., Bradley, B.P., DeBono, J., Painter, M., 1997. Time course of attentional bias for threat information in non-clinical anxiety. Behav. Res. Ther. 35, 297-303.

Mogg, K., Bradley, B.P., 2002. Selective orienting of attention to masked threat faces in social anxiety. Behav. Res. Ther. 40, 14031414.

Mogg, K., Philippot, P., Bradley, B.P., 2004. Selective attention to angry faces in clinical social phobia. J. Abnorm. Psychol. 113, $160-165$.

Moser, J.S., Huppert, J.D., Duval, E., Simons, R.F., 2008. Face processing biases in social anxiety: an electrophysiological study. Biol. Psychol. 78, 93-103.

Musa, C., Lepine, J.P., Clark, D.M., Mansell, W., Ehlers, A., 2003. Selective attention in social phobia and the moderating effect of a concurrent depressive disorder. Behav. Res. Ther. 41, 10431054.

Oei, N.Y.L., Tollenaar, M.S., Spinhoven, P., Elzinga, B.M., 2009. Hydrocortisone reduces emotional distracter interference in working memory. Psychoneuroendocrinology, doi:10.1016/j. psyneuen.2009.03.015.

Picton, T.W., Bentin, S., Berg, P., Donchin, E., Hillyard, S.A., Johnson, R., Miller, G.A., Ritter, W., Ruchkin, S.S., Rugg, M.D., Taylor, M.J., 2000. Guidelines for using human event-related potentials to study cognition: recording standards and publication criteria. Psychophysiology 37, 127-152.

Putman, P., Hermans, E., van Honk, J., 2004. Emotional Stroop performance for masked angry faces: it's BAS, not BIS. Emotion 4, 305-311.

Putman, P., Hermans, E.J., van Honk, J., 2007a. Exogenous cortisol shifts a motivated bias from fear to anger in spatial working memory for facial expressions. Psychoneuroendocrinology 32 , $14-21$.

Putman, P., Hermans, E.J., Koppeschaar, H., Van Schijndel, A., van Honk, J., 2007b. A single administration of cortisol acutely reduces preconscious attention for fear in anxious young men. Psychoneuroendocrinology 32, 793-802.

Rapee, R.M., Heimberg, R.G., 1997. A cognitive-behavioral model of anxiety in social phobia. Behav. Res. Ther. 35, 741756.

Roelofs, K., van Peer, J., Berretty, E., de Jong, P., Spinhoven, P., Elzinga, B.M., 2009. HPA-axis hyperresponsiveness is associated with increased social avoidance behavior in social phobia. Biol. Psychiatry 65, 336-343.

Roelofs, K., Elzinga, B.M., Rotteveel, M., 2005. The effects of stressinduced cortisol responses on approach-avoidance behavior. Psychoneuroendocrinology 30, 665-677.

Rossignol, M., Anselme, C., Vermeulen, N., Philippot, P., Campanella, S., 2007. Categorical perception of anger and disgust facial expression is affected by non-clinical social anxiety: an ERP study. Brain Res. 1132, 166-176.

Sapolsky, R.M., Romero, L.M., Munck, A.U., 2000. How do glucocorticoids influence stress responses? Integrating permissive, suppressive, stimulatory, and preparative actions. Endocr. Rev. 21, $55-89$.

Schultz, L.T., Heimberg, R.G., 2008. Attentional focus in social anxiety disorder: potential for interactive processes. Clin. Psychol. Rev. 28, 1206-1221. 
Schupp, H.T., Öhman, A., Junghofer, M., Weike, A.I., Stockburger, J., Hamm, A.O., 2004. The facilitated processing of threatening faces: an ERP analysis. Emotion 4, 189-200.

Soravia, L.M., Heinrichs, M., Aerni, A., Maroni, C., Schelling, G., Ehlert, U., Roozendaal, B., De Quervain, D.J.F., 2006. Glucocorticoids reduce phobic fear in humans. Proc. Natl. Acad. Sci. U.S.A. 103, 5585-5590.

Spector, I.P., Pecknold, J.C., Libman, E., 2003. Selective attentional bias related to the noticeability aspect of anxiety symptoms in generalized social phobia. J. Anxiety Disord. 17, 517-531.

Spielberger, C.D., 1983. Manual for the State-Trait Anxiety Inventory (STAl-form Y). Consulting Psychologists Press, Palo Alto, CA.

Turner, S.M., Beidel, D.C., Dancu, C.V., Stanley, M.A., 1989. An empirically derived inventory to measure social fears and anxiety: the social phobia and anxiety inventory. Psychol. Assess. 1, 35-40. van Aken, M.O., Romijn, J.A., Miltenburg, J.A., Lentjes, E.G.W.M., 2003. Automated measurement of salivary cortisol. Clin. Chem. 49, 1408-1409.

van Honk, J., Tuiten, A., van den Hout, M., Koppeschaar, H., Thijssen, J., de Haan, E., Verbaten, R., 1998. Baseline salivary cortisol levels and preconscious selective attention for threat. A pilot study. Psychoneuroendocrinology 23, 741-747. van Honk, J., Tuiten, A., van den Hout, M., Koppeschaar, H., Thijssen, J., de Haan, E., Verbaten, R., 2000. Conscious and preconscious selective attention to social threat: different neuroendocrine response patterns. Psychoneuroendocrinology 25, 577-591.

van Hooff, J.C., Dietz, K.C., Sharma, D., Bowman, H., 2008. Neural correlates of intrusion of emotion words in a modified Stroop task. Int. J. Psychophysiol. 67, 23-34.

van Peer, J.M., Roelofs, K., Rotteveel, M., van Dijk, J.G., Spinhoven, P., Ridderinkhof, K.R., 2007. The effects of cortisol administration on approach-avoidance behavior: an event-related potential study. Biol. Psychol. 76, 135-146.

van Peer, J.M., Spinhoven, P., van Dijk, J.G., Roelofs, K., 2009. Cortisol-induced enhancement of emotional face processing in social phobia depends on symptom severity and motivational context. Biol. Psychol. 81, 123-130.

Williams, J.M.G., Mathews, A., Macleod, C., 1996. The emotional Stroop task and psychopathology. Psychol. Bull. 120, 324.

Williams, L.M., Palmer, D., Liddell, B.J., Song, L., Gordon, E., 2006. The 'when' and 'where' of perceiving signals of threat versus nonthreat. Neuroimage 31, 458-467. 